\title{
Eicosapentaenoic Acid-enriched Nutritional Supplement
}

\author{
National Cancer Institute
}

\section{Source}

National Cancer Institute. Eicosapentaenoic Acid-enriched Nutritional Supplement. NCI

Thesaurus. Code C120042.

A nutritional supplement enriched with eicosapentaenoic acid (EPA), which is an essential, polyunsaturated, 20-carbon omega-3 fatty acid found in fish oil, with potential antiinflammatory and anti-cachectic activities. Upon oral intake of the EPA-enriched nutritional supplement, EPA is incorporated in cell membrane phospholipids and replaces arachidonic acid. This affects the production of pro-inflammatory mediators, such as tumor necrosis factor-alpha (TNF-a), interleukin-1 (IL-1) and IL-6, through the inhibition of nuclear factor kappa B (NF-kB) activity. This inhibits inflammation and may abrog ate the cachexia-mediated decrease of lean body mass (LBM), which may lead to increased body weight. Pro-inflammatory mediators, such as TNF-a, interferon-gamma, and certain interleukins, such as IL- 6 and IL-1 b, play a key role in cachexia. 